UNIVERSITÀ DEGLI STUDI DI BERGAMO

DIPARTIMENTO DI INGEGNERIA DELL'INFORMAZIONE

E METODI MATEMATICI

QUADERNI DEL DIPARTIMENTO

Department of Information Technology and Mathematical Methods

Working Paper

Series "Mathematics and Statistics"

n. $3 / \mathrm{MS}-2010$

Poisson Pencils, Algebraic Integrability,

and Separation of Variables

by

G. Falqui, M. Pedroni 
COMITATO DI REDAZIONE ${ }^{\S}$

Series Information Technology (IT): Stefano Paraboschi

Series Mathematics and Statistics (MS): Luca Brandolini, Ilia Negri

\footnotetext{
$\S$ L'accesso alle Series è approvato dal Comitato di Redazione. I Working Papers della Collana dei Quaderni del Dipartimento di Ingegneria dell'Informazione e Metodi Matematici costituiscono un servizio atto a fornire la tempestiva divulgazione dei risultati dell'attività di ricerca, siano essi in forma provvisoria o definitiva.
} 


\title{
Poisson Pencils, Algebraic Integrability, and Separation of Variables
}

\author{
Gregorio Falqui $^{1}$ and Marco Pedroni ${ }^{2}$ \\ 1 Dipartimento di Matematica e Applicazioni, Università di Milano-Bicocca, Via Roberto Cozzi 53, \\ I-20125 Milano, Italy \\ ${ }^{2}$ Dipartimento di Ingegneria dell'Informazione e Metodi Matematici, Università di Bergamo, Viale \\ Marconi 5, I-24044 Dalmine (BG), Italy \\ E-mail addresses: \\ gregorio.falqui@unimib.it, marco.pedroni@unibg.it
}

May 11, 2010

\begin{abstract}
In this paper we review a recently introduced method for solving the HamiltonJacobi equations by the method of Separation of Variables. This method is based on the notion of pencil of Poisson brackets and on the bihamiltonian approach to integrable systems. We discuss how separability conditions can be intrinsically characterized within such a geometrical set-up, the definition of the separation coordinates being encompassed in the bihamiltonian structure itself. We finally discuss these constructions studying in details a particular example, based on a generalization of the classical Toda Lattice.
\end{abstract}

\section{Contents}

1 Introduction 2

2 Some issues in the geometry of bihamiltonian manifolds 5

2.1 Geometry of regular bihamiltonian manifolds and Darboux-Nijenhuis coordinates . . . . . . . . . . . . . . . . . 8

2.2 On Darboux-Nijenhuis coordinates . . . . . . . . . . . . . . . 10

3 Separability conditions in the bihamiltonian setting 12

4 Transversal distributions and separation relations 16

5 Example: a generalized Toda Lattice $\quad 17$

5.1 Separation of Variables . . . . . . . . . . . . . . . . . . . . . . 20

5.2 A remarkable subsystem: the open $\mathrm{Toda}_{3}^{4}$ system . . . . . . . . . . 23 


\section{Introduction}

The study of the separability of the Hamilton-Jacobi (HJ) equations associated with a given Hamiltonian function $H$ is a very classical issue in Mechanics, dating back to the foundational works of Jacobi, Stäckel, Levi-Civita, and others. It has recently received a strong renewed interest thanks to its applications to the theory of integrable PDEs of $\mathrm{KdV}$ type (namely, the theory of finite gap integration) and to the theory of quantum integrable systems (see, e.g., [14, 41]).

As it is well known, the problem can be formulated as follows. Let $(M, \omega)$ be a $2 n$ dimensional symplectic manifold, and let $\left(p_{1}, \ldots, p_{n}, q_{1}, \ldots, q_{n}\right) \equiv(\mathbf{p}, \mathbf{q})$ be canonical coordinates in $U \subset M$, i.e., $\omega_{\left.\right|_{U}}=\sum_{i=1}^{n} d p_{i} \wedge d q_{i}$. The (stationary) HJ equation reads

$$
H\left(q_{1}, \ldots, q_{n} ; \frac{\partial S}{\partial q_{1}}, \ldots, \frac{\partial S}{\partial q_{n}}\right)=E
$$

Definition 1.1 A complete integral $S\left(\mathbf{q} ; \alpha_{1}, \ldots, \alpha_{n}\right)$ of the HJ equation is a solution of (1.1), depending on $n$ parameters $\left(\alpha_{1}, \ldots, \alpha_{n}\right)$ such that Det $\frac{\partial^{2} S}{\partial q_{i} \partial \alpha_{j}} \neq 0$. The Hamiltonian $H$ is said to be separable in the coordinates $(\mathbf{p}, \mathbf{q})$ if the HJ equation admits an additively separated complete integral, that is, a complete integral of the form

$$
S\left(\mathbf{q} ; \alpha_{1}, \ldots, \alpha_{n}\right)=\sum_{i=1}^{n} S_{i}\left(q_{i} ; \alpha_{1}, \ldots, \alpha_{n}\right) .
$$

In this paper we will focus on an equivalent definition of separability, originally due to Jacobi and recently widely used by Sklyanin and his collaborators. Let us consider an integrable Hamiltonian $H$, that is, let us suppose that, along with $H=H_{1}$ we have further $(n-1)$ mutually commuting integrals of the motion $H_{2}, \ldots H_{n}$, with $d H_{1} \wedge \ldots \wedge$ $d H_{n} \neq 0$.

Definition 1.2 An integrable system $\left(H_{1}, \ldots, H_{n}\right)$ is separable in the coordinates $(\mathbf{p}, \mathbf{q})$ if there exist $n$ non-trivial relations

$$
\Phi_{i}\left(q_{i}, p_{i} ; H_{1}, \ldots, H_{n}\right)=0, \quad i=1, \ldots n
$$

connecting single pairs $\left(q_{i}, p_{i}\right)$ of canonical coordinates with the $n$ Hamiltonians $H_{i}$.

This alternative definition is indeed a constructive approach to separability, since the knowledge of the separation relations (1.3) allows one to reduce the problem of finding a separated solution of HJ to quadratures. In fact, let us suppose that the relations $\Phi_{i}\left(q_{i}, p_{i} ; H_{1}, \ldots, H_{n}\right)=0$, for $i=1, \ldots, n$, can be solved in terms of the $p_{i}$ to get $p_{i}=p_{i}\left(q_{i} ; H_{1}, \ldots, H_{n}\right)$. Then one can define:

$$
S\left(\mathbf{q} ; \alpha_{1}, \ldots, \alpha_{n}\right)=\left.\sum_{i=1}^{n} \int^{q_{i}} p_{i}\left(q_{i}^{\prime} ; H_{1}, \ldots, H_{n}\right)\right|_{H_{i}=\alpha_{i}} d q_{i}^{\prime} .
$$


This is by construction a separated solution of HJ; the fact that it is a complete integral is equivalent to the (already assumed) fact that the integrals of the motion depend non trivially on the momenta.

In intrinsic terms, one notices that the equations $H_{i}=\alpha_{i}$, for $i=1, \ldots, n$, define a foliation $\mathcal{F}$ of $M$. The leaves of $\mathcal{F}$ are nothing but the (generalized) tori of the Arnol'dLiouville theorem. The foliation is Lagrangian, that is, the restriction of the two-form $\omega$ to $\mathcal{F}$ vanishes. Hence the restriction to $\mathcal{F}$ of the Liouville form $\theta=\sum_{i=1}^{n} p_{i} d q_{i}$ is (locally) exact. Indeed, the function $S$ defined by (1.4) is a (local) potential for such restriction. What is non intrinsic, and singles out the separation coordinates $(\mathbf{p}, \mathbf{q})$, is that the separation relations (1.3), which are another set of defining equations for the foliation $\mathcal{F}$, have the very special property of containing a single pair of canonical coordinates at a time. The problem to find such a system of coordinates and relations is the core of the theory of SoV. In particular, a natural question arises:

Is it possible to formulate intrinsic condition(s) on the Hamiltonians $\left(H_{1}, \ldots, H_{n}\right)$ to a priori ensure separability in a (given) set of canonical coordinates?

Actually, this is the main issue studied by both the 'classical' Eisenhart-Benenti theory [6] of separability of natural systems defined on cotangent bundles to Riemannian manifolds $(M, g)$, as well as the 'modern' theory, mainly due to the St. Petersburg [41] and Montreal [1, 26] schools, of SoV for systems admitting a Lax representation. We notice that both such general approaches require the presence of an additional structure to solve the problem. Indeed, the Eisenhart-Benenti theory requires the existence of a conformal Killing tensor for the metric $g$, while the Lax theory requires - in addition to the knowledge of a Lax representation with spectral parameter for the Hamiltonian system under study - the existence of an $r$-matrix structure for such a Lax representation.

The method we review in this paper has recently been exposed in the literature (see, e.g., $[9,37,7,17,8,27,16,38,39,21,5,18,19]$ ), and can be seen as a kind of bridge between the classical and the modern points of view, putting an emphasis on the geometrical aspects of the Hamiltonian theory. Its 'additional' structure is simply the requirement of the existence, on the symplectic manifold $(M, \omega)$, of a second Hamiltonian structure, compatible with the one defined by $\omega$. Namely, the bihamiltonian structure on $M$ will allow us:

1. To encompass the definition of a special set of coordinates, to be called DarbouxNijenhuis $(D N)$ coordinates, within a well defined geometrical object.

2. To formulate intrinsic (i.e., tensorial) conditions for the separability of a Hamiltonian integrable system, in the DN coordinates associated with the bihamiltonian structure.

3. To give recipes to characterize, find and handle sets of DN coordinates.

A very important issue that is close to the separability problem is the notion of algebraically completely integrable Hamiltonian systems (see, e.g., [3]). In general, a (Hamiltonian) system is said to be algebraically integrable whenever its flow(s) linearize 
on the Jacobian variety of an algebraic curve (the spectral curve). The latter is usually recovered as the characteristic polynomial of the Lax matrix of the system (provided the latter is known/given), and the integration of the equation of motion reconducted to a Jacobi inversion problem.

It is fair to say that in the bihamiltonian setting we are herewith discussing we are, so far, not able to provide general criteria for the algebraic integrability of our systems. However, as we shall see below, we are in a position to make contact with the problem of algebraic integrability, at least in the (slightly different and weaker) setting of Veselov and Novikov [44], that can be summarized as follows.

Given a Hamiltonian systems one assumes that the phase space $M$ fulfills the following properties:

a) $M$ has the fibered structure

$$
M \stackrel{S^{k} \Gamma}{\longrightarrow} B
$$

where the base $B$ is an $n$-dimensional manifold whose points $b$ determine an algebraic curve $\Gamma(b)$, and the fiber is the $k$-th symmetric product of that curve. In more details, one requires that $\Gamma(b)$ be given as an $m$-sheeted covering $\Gamma(b) \stackrel{m}{\longrightarrow} \mathbb{C}$ of the complex $\lambda$-plane, and that points of $M$ can be parameterized via the curve $\Gamma(b)$, and a set of $k$ points on it, that is, the coordinates $\lambda_{1}, \ldots, \lambda_{k}$ of the projection on the $\lambda$-plane of a set of points on it, as well as discrete parameters $\epsilon_{i}$ that specify on which sheet of the covering the points live.

b) An Abelian differential $Q(\Gamma)$ on $\Gamma$ (or possibly on a covering of $\Gamma$ ), smoothly depending on the point $b \in B$, is defined. It is furthermore required that, if $Q(\Gamma)$ is given by

$$
Q(\Gamma)=Q(b ; \lambda) d \lambda
$$

according to the representation of $\Gamma$ as a covering of the $\lambda$-plane, the closed twoform

$$
\omega_{Q}=\sum_{i=1}^{k} d Q\left(b ; \lambda_{i}\right) \wedge d \lambda_{i}
$$

gives rise to a Poisson bracket, conveniently called algebro-geometric Poisson bracket, with $\lambda_{i}$ and $\mu_{i}=Q\left(b ; \lambda_{i}\right)$ playing the role of Darboux coordinates on the symplectic leaves of this bracket.

In such a case, it was proven in [44] that functions that depend only on the curve $\Gamma$ - i.e., on the points of $B$ - are in involution with respect to the Poisson bracket defined by (1.7), and these geometric data explicitly define action-angle variables for the corresponding Hamiltonian flows. Moreover, Sklyanin's method [41] of the poles of the Baker-Akhiezer function can be seen as a particularly efficient scheme of implementing the Veselov-Novikov axiomatic picture. In [22] we studied some relations between the bihamiltonian approach to SoV and the Veselov-Novikov description of algebraic integrability, especially within the example of the Volterra lattice. 
As far as the organization of this paper is concerned, in Section 2 we briefly introduce the notions of bihamiltonian geometry relevant for the subsequent sections. In particular, we discuss the notion of DN coordinates, as well as methods to find them. In Section 3 we present the main theorems of the bihamiltonian set-up for SoV, namely, the tensorial conditions ensuring separability of the HJ equations in DN coordinates. Section 4 is devoted to separable systems coming from bihamiltonian systems by means of a reduction along a suitable transversal distribution. Then we discuss our constructions in a specific example, whose separability, to the best of our knowledge, has not been considered in the literature yet. It is a generalization of the periodic Toda lattice with four sites. In Section 5 we recall its definition, and show how the "bihamiltonian recipe" for SoV can be applied to it. Although our constructions can be generalized to the generic $N$-site system, for the sake of concreteness and brevity we choose to consider the four-site system only, and sometimes rely on direct computations to prove some of its properties. In the last subsection we apply our geometrical scheme to study a specific reduction of this generalized Toda system, and to find integrals of the motion which are not encompassed in the Lax representation. This result can possibly be a suitable step towards an alternative approach to the so-called chopping method [12] for the full (non-periodic) Toda Lattice.

\section{Some issues in the geometry of bihamiltonian man- ifolds}

We start this section recalling some well known facts in the theory of Poisson manifolds (see, e.g., [43]).

Definition 2.1 A Poisson manifold $(M,\{\cdot, \cdot\})$ is a manifold endowed with a Poisson bracket, that is, a bilinear antisymmetric composition laws defined on the space $C^{\infty}(M)$ satisfying:

1. The Leibniz rule: $\{f g, h\}=f\{g, h\}+g\{f, h\}$;

2. The Jacobi identity: $\{f,\{g, h\}\}+\{g,\{h, f\}\}+\{h,\{f, g\}\}=0$.

A Poisson bracket (or Poisson structure) can be equivalently described with the corresponding Poisson tensor, i.e., with the application $P: T^{*} M \rightarrow T M$, smoothly varying with $m \in M$, defined by

$$
\{f, g\}=\langle d f, P d g\rangle,
$$

where $\langle\cdot, \cdot\rangle$ denotes the canonical pairing between $T^{*} M$ and $T M$. In a given coordinate system $\left(x^{1}, \ldots, x^{n}\right)$ on $M$, the Poisson tensor $P$ associated with the Poisson bracket $\{\cdot, \cdot\}$ is represented as

$$
P=\sum_{i, j=1}^{n} P^{i j} \frac{\partial}{\partial x_{i}} \wedge \frac{\partial}{\partial x_{j}}, \quad \text { with } P^{i j}=\left\{x^{i}, x^{j}\right\} .
$$


The Jacobi identity is translated into a quadratic differential condition on the matrix $\left(P^{i j}\right)$, known as the vanishing of the Schouten bracket, which in local coordinates reads

$$
\sum_{s=1}^{n}\left(P^{i s} \frac{\partial P^{j k}}{\partial x^{s}}+P^{j s} \frac{\partial P^{k i}}{\partial x^{s}}+P^{k s} \frac{\partial P^{i j}}{\partial x^{s}}\right)=0, \quad \forall i>j>k .
$$

A function in $C^{\infty}(M)$ is said to be a Casimir function if its Poisson bracket with any other function on $M$ vanishes, or, equivalently, if its differential lies in the kernel of $P$.

The local structure of a Poisson manifold is described in details in $[45,14,43]$. For our purposes, we just need to recall that (in the open subset of $M$ where the rank $r=2 n$ of the Poisson tensor is maximal) $M$ is foliated in symplectic leaves, that (locally) are the common level sets of $k$ Casimir functions $C_{1}, \ldots, C_{k}$ of $P$. The dimension of $M$ is related with the integers $n$ and $k$ by $\operatorname{dim} M=k+2 n$.

Let us now come to the definition of bihamiltonian manifold.

Definition 2.2 A manifold $M$ is called a bihamiltonian manifold if it is endowed with two Poisson brackets $\{\cdot, \cdot\}$ and $\{\cdot, \cdot\}^{\prime}$ such that, for any $\lambda \in \mathbb{R}$ (or $\lambda \in \mathbb{C}$ if $M$ is complex), the linear combination

$$
\{f, g\}^{\prime}-\lambda\{f, g\} \equiv\left\langle d f,\left(P^{\prime}-\lambda P\right) d g\right\rangle
$$

defines a Poisson bracket. This property is known as the compatibility condition between the two brackets.

The expression (2.2) will be referred to as pencil of Poisson brackets, and the sum $P_{\lambda}=P^{\prime}-\lambda P$ as pencil of Poisson tensors. The most 'popular' property of bihamiltonian manifolds is contained in the following

Proposition 2.3 Let $f$ and $f^{\prime}$ be two functions on a bihamiltonian manifold $M$, which satisfy the characteristic condition $P d f=P^{\prime} d f^{\prime}$. Then the Poisson brackets $\left\{f, f^{\prime}\right\}$ and $\left\{f, f^{\prime}\right\}^{\prime}$ vanish.

Proof. It consists of a one-line computation. Let us consider, e.g., $\left\{f, f^{\prime}\right\}$ :

$$
\left\{f, f^{\prime}\right\}=\left\langle d f, P d f^{\prime}\right\rangle=-\left\langle d f^{\prime}, P d f\right\rangle=-\left\langle d f^{\prime}, P^{\prime} d f^{\prime}\right\rangle=0 .
$$

The vanishing of the other Poisson bracket is even easier.

Definition 2.4 A vector field $X$ that can be written as $X=P d f=P^{\prime} d f^{\prime}$ is called a bihamiltonian vector field.

Corollary 2.5 Let $f_{i}$, with $i \in \mathbb{Z}$, be a sequence of functions satisfying

$$
P d f_{i}=P^{\prime} d f_{i-1}
$$

Then $\left\{f_{i}, f_{k}\right\}=\left\{f_{i}, f_{k}\right\}^{\prime}=0$ for all $i, k \in \mathbb{Z}$. 
Proof. Using twice equation (2.3) and the antisymmetry of the Poisson brackets we have

$$
\begin{aligned}
\left\{f_{i}, f_{k}\right\} & =\left\langle d f_{i}, P d f_{k}\right\rangle=\left\langle d f_{i}, P^{\prime} d f_{k-1}\right\rangle=-\left\langle d f_{k-1}, P^{\prime} d f_{i}\right\rangle \\
& =-\left\langle d f_{k-1}, P d f_{i+1}\right\rangle=\left\langle d f_{i+1}, P d f_{k-1}\right\rangle=\left\{f_{i+1}, f_{k-1}\right\} .
\end{aligned}
$$

Supposing $k>i$ and iterating this procedure $(k-i)$ times, we get $\left\{f_{i}, f_{k}\right\}=\left\{f_{k}, f_{i}\right\}$, so that $\left\{f_{i}, f_{k}\right\}=0$. The vanishing of $\left\{f_{i}, f_{k}\right\}^{\prime}$ is an easy consequence.

Using the same technique, we can prove

Amplification 2.6 Let $\left\{f_{n}\right\}_{n \geq 0}$ and $\left\{g_{n}\right\}_{n \geq 0}$ be two sequences of functions satisfying

$$
P d f_{i}=P^{\prime} d f_{i-1}, P d f_{0}=0 ; \quad P d g_{i}=P^{\prime} d g_{i-1}, P d g_{0}=0 .
$$

Then, along with $\left\{f_{n}, f_{m}\right\}=\left\{f_{n}, f_{m}\right\}^{\prime}=\left\{g_{n}, g_{m}\right\}=\left\{g_{n}, g_{m}\right\}^{\prime}=0$, it holds

$$
\left\{f_{n}, g_{m}\right\}=\left\{f_{n}, g_{m}\right\}^{\prime}=0 \quad \forall n, m \geq 0 .
$$

The family of vector fields associated with a sequence of functions satisfying the recursion relations (2.3) are customarily said to form a Lenard-Magri sequence. Those sequences starting from the null vector field, as in Amplification 2.6, are pictorially called anchored Lenard-Magri sequences. Notice that anchored Lenard sequences can occur in bihamiltonian manifold where at least one of the Poisson brackets is non-symplectic (indeed, e.g., $d f_{0}$ is a non-trivial element of the kernel of $P$ ). We can compactly express equations (2.4) relative, say, to the sequence $f_{i}$ by considering the formal Laurent series $f(\lambda)=\sum_{i=0}^{\infty} f_{i} / \lambda^{i}$ and writing the equation

$$
\left(P^{\prime}-\lambda P\right) d f(\lambda)=0 .
$$

If, as it often happens in the applications, inside the family $f_{i}$ we have an element $f_{n}$ satisfying $P^{\prime} d f_{n}=0$, we can form a polynomial Casimir of the pencil as

$$
F(\lambda)=\lambda^{n} f_{0}+\lambda^{n-1} f_{1}+\cdots+f_{n}
$$

In analogy with the definition of Casimir of a Poisson bracket, Laurent series satisfying (2.5) are called Casimirs of the Poisson pencil. The reader should, however, bear in mind that while Casimir functions for a single Poisson bracket are, in a sense, uninteresting functions, Casimirs of a pencil of Poisson bracket compactly encode non-trivial dynamics and constants of the motion. More precisely, anchored Lenard sequences may give rise to families of integrable systems. Let us see how this happens in the case of a $(2 n+1)$-dimensional manifold endowed with a rank- $2 n$ pencil of Poisson tensors. Let us suppose that we have found a polynomial Casimir of the form (2.6), such that the 
$(n+1)$ functions $f_{0}, \ldots, f_{n}$ are independent. Let $\mathcal{S}_{c}$ be a generic symplectic leaf of $P$, corresponding to $f_{0}=c$. The vector fields $X_{f_{i}}$, with $i=1, \ldots, n$, are tangent to $\mathcal{S}_{c}$, are Hamiltonian on $\mathcal{S}_{c}$ (with respect to the symplectic form given by the restriction of $P$ ), and the restrictions of the functions $f_{1}, \ldots, f_{n}$ provide $n$ commuting integrals for each of them. In general, it holds [23, 24]:

Proposition 2.7 Let $\left(M, P, P^{\prime}\right)$ be a bihamiltonian manifold of dimension $d=2 n+$ $k$, and let $\operatorname{dim}\left(\operatorname{Ker}\left(P^{\prime}-\lambda P\right)\right)=k$ for generic values of $\lambda$. Let us suppose that $H^{(1)}(\lambda), \ldots, H^{(k)}(\lambda)$ are $k$ polynomial Casimirs of the pencil $P_{\lambda}$ of the form

$$
H^{(a)}(\lambda)=\lambda^{n_{a}} H_{0}^{(a)}+\lambda^{n_{a}-1} H_{1}^{(a)}+\cdots+H_{n_{a}}^{(a)},
$$

such that the collection of differentials $\left\{d H_{j}^{(a)}\right\}_{j=0, \ldots, n_{a}}^{a=1, \ldots, k}$ defines a $(k+n)$-dimensional distribution in $T^{*} M$. Then the vector fields defined by the anchored sequences associated with the $H^{(a)}$ are integrable (in the Arnol'd-Liouville sense) on the generic symplectic leaves of $P$.

\subsection{Geometry of regular bihamiltonian manifolds and Darboux- Nijenhuis coordinates}

An important class of bihamiltonian manifold occurs when an element of the Poisson pencil (which without loss of generality we will assume to be $P$ ) is everywhere invertible, i.e., the Poisson bracket $\{\cdot, \cdot\}$ associated with $P$ is symplectic. The possibility of defining the inverse to one of the Poisson tensors leads us to introduce a fundamental object in the bihamiltonian theory of SoV: the Nijenhuis (or Hereditary, or Recursion) operator

$$
N=P^{\prime} P^{-1},
$$

together with its transpose $N^{*}=P^{-1} P^{\prime}$. By definition, $N$ (resp., $N^{*}$ ) is an endomorphism of the tangent bundle to $M$ (resp., of the cotangent bundle). As a remarkable consequence of the compatibility between $P$ and $P^{\prime}$, the Nijenhuis torsion of $N$, defined by its action on a pair of vector fields $X, Y$ as

$$
T(N)(X, Y)=[N X, N Y]-N([N X, Y]+[X, N Y]-N[X, Y]),
$$

identically vanishes [33]. So, from the classical Frölicher-Nijenhuis theory, we know that its eigenspaces are integrable distributions. Such distributions will be the building blocks of the bihamiltonian set-up for SoV.

To explain this point, we have to make some remarks and a genericity assumption. It can be shown that, owing to the antisymmetry of the Poisson tensors defining $N$, the eigenspaces of $N$ are pointwise even dimensional. Throughout this paper, we will assume that, for generic points $m \in M$, the operator $N_{m}$ has the maximal number $n=\frac{1}{2} \operatorname{dim} M$ of different eigenvalues $\lambda_{1}, \ldots, \lambda_{n}$, so that the dimension of the eigenspace relative to any eigenvalue is 2 . Otherwise stated, the characteristic polynomial of $N$ is the square 
of its degree- $n$ minimal polynomial $\Delta_{N}(\lambda)$, whose roots are pairwise distinct. We will call regular a bihamiltonian manifolds endowed with a Poisson pencil with at least one of the elements of the Poisson pencil invertible, and such that the eigenvalues of the associated Nijenhuis tensor are maximally distinct.

Theorem 2.8 On a regular bihamiltonian manifold there exists a class of coordinates $\left(y_{i}, x_{i}\right)$, to be called Darboux-Nijenhuis (DN) coordinates, satisfying the two properties:

(Darboux) They are canonical, that is, $\left\{x_{i}, y_{j}\right\}=\delta_{i j},\left\{x_{i}, x_{j}\right\}=\left\{y_{i}, y_{j}\right\}=0$.

(Nijenhuis) They diagonalize $N$, that is, $N=\sum_{i} \lambda_{i}\left(\frac{\partial}{\partial y_{i}} \otimes d y_{i}+\frac{\partial}{\partial x_{i}} \otimes d x_{i}\right)$.

The proof of this theorem can be found in [30, 23]. Here we will sketch it and discuss its meaning. In words, the assertion states that DN coordinates are defined by the spectral properties of $N$, as follows. For all $m$ in the open set $U$ where the eigenvalues $\lambda_{i}$ of $N$ (which are the same as the eigenvalues of $N^{*}$ ) satisfy $\lambda_{i} \neq \lambda_{j}$ for $i \neq j$, the cotangent space $T_{m}^{*} M$ admits the decomposition

$$
T_{m}^{*} M=\oplus_{i=1}^{n} \mathcal{D}_{m, \lambda_{i}}, \quad \operatorname{dim} \mathcal{D}_{m, \lambda_{i}}=2,
$$

into eigenspaces of $N^{*}$. Thanks to the vanishing of the torsion of $N$, each eigenspace $\mathcal{D}_{m, \lambda_{i}}$ is locally generated by differentials of pairs of independent functions $\left(f_{i}, g_{i}\right)$. This means that the pointwise decomposition (2.9) holds (in $U^{\prime} \subset U$ ) as

$$
T^{*} M_{\left.\right|_{U^{\prime}}}=\oplus_{i=1}^{n} \mathcal{D}_{\lambda_{i}}
$$

where $\mathcal{D}_{\lambda_{i}}$ is spanned by $d f_{i}$ and $d g_{i}$, with $N^{*} d f_{i}=\lambda_{i} d f_{i}$ and $N^{*} d g_{i}=\lambda_{i} d g_{i}$.

Functions whose differential belong to different summands $\mathcal{D}_{\lambda_{i}}$ are in involution with respect to the Poisson brackets defined both by $P$ and $P^{\prime}$. Indeed, suppose that $f_{1}$ and $f_{2}$ satisfy $N^{*} d f_{1}=\lambda_{1} d f_{1}$ and $N^{*} d f_{2}=\lambda_{2} d f_{2}$, with $\lambda_{1} \neq \lambda_{2}$. The relation $N^{*}=P^{-1} P^{\prime}$ implies that $P^{\prime} d f_{1}=\lambda_{1} P d f_{1}$ and $P^{\prime} d f_{2}=\lambda_{2} P d f_{2}$. So,

$$
\left\{f_{1}, f_{2}\right\}^{\prime}=\left\{\begin{array}{l}
\left\langle d f_{1}, P^{\prime} d f_{2}\right\rangle=\lambda_{2}\left\langle d f_{1}, P d f_{2}\right\rangle=\lambda_{2}\left\{f_{1}, f_{2}\right\} \\
-\left\langle d f_{2}, P^{\prime} d f_{1}\right\rangle=-\lambda_{1}\left\langle d f_{2}, P d f_{1}\right\rangle=\lambda_{1}\left\{f_{1}, f_{2}\right\}
\end{array}\right.
$$

whence the assertion. It is equally straightforward to realize that the only non vanishing Poisson brackets have the form

$$
\left\{f_{i}, g_{i}\right\}=F_{i}\left(f_{i}, g_{i}\right), \quad\left\{f_{i}, g_{i}\right\}^{\prime}=F_{i}^{\prime}\left(f_{i}, g_{i}\right), \quad i=1, \ldots, n .
$$

This means that from the $n$ pairs of functions $\left(f_{i}, g_{i}\right)$ we can construct by quadratures a set of canonical coordinates satisfying the Nijenhuis property of Theorem 2.8. Thus the class of coordinates where to frame the bihamiltonian set-up for SoV admits a clearcut and simple geometrical description. Admittedly, in the general case the computation of DN coordinates requires the integration of the two-dimensional distributions $\mathcal{D}_{\lambda_{i}}$ associated with the eigenvalues $\lambda_{i}$ of $N^{*}$. Fortunately enough, there are instances (that frequently occur in the applications) in which DN coordinates can be found in an easier way.

For an analysis of Darboux-Nijenhuis coordinates within the theory of multi-hamiltonian structure on loop algebras, see [17, 11, 25]. 


\subsection{On Darboux-Nijenhuis coordinates}

In this subsection we will briefly discuss conditions and 'recipes' to algebraically find and/or characterize sets of Darboux-Nijenhuis coordinates on regular bihamiltonian manifolds. A very simplifying instance occurs whenever the eigenvalues $\lambda_{i}$ of $N$ (that are, in general, functions of the point $m \in M)$ are functionally independent. It holds (see, e.g., [32]):

Proposition 2.9 Let us define $I_{k}=\frac{1}{2 k} \operatorname{Tr} N^{k}$ for $k=1, \ldots, n$. In the open set $U$ where $d I_{1} \wedge \cdots \wedge d I_{n} \neq 0$ the eigenvalues $\lambda_{i}, i=1, \ldots, n$, are functionally independent, satisfy $N^{*} d \lambda_{i}=\lambda_{i} d \lambda_{i}$, and so may be used to construct a set of Darboux-Nijenhuis coordinates.

Proof. We express the normalized traces $I_{k}$ of the Nijenhuis tensor $N$ in terms of its eigenvalues as $k I_{k}=\sum_{i=1}^{n} \lambda_{i}^{k}$. Hence $d I_{k}=\sum_{i=1}^{n} \lambda_{i}^{k-1} d \lambda_{i}$, that is, in matrix terms:

$$
\left[\begin{array}{c}
d I_{1} \\
d I_{2} \\
\vdots \\
d I_{n}
\end{array}\right]=\left[\begin{array}{cccc}
1 & 1 & \cdots & 1 \\
\lambda_{1} & \lambda_{2} & \cdots & \lambda_{n} \\
\vdots & \vdots & \ddots & \vdots \\
\lambda_{1}^{n-1} & \lambda_{2}^{n-1} & \cdots & \lambda_{n}^{n-1}
\end{array}\right] \cdot\left[\begin{array}{c}
d \lambda_{1} \\
d \lambda_{2} \\
\vdots \\
d \lambda_{n}
\end{array}\right]
$$

So we have

$$
d I_{1} \wedge \cdots \wedge d I_{n}=\left(\prod_{i \neq j}\left(\lambda_{i}-\lambda_{j}\right)\right) d \lambda_{1} \wedge \cdots \wedge d \lambda_{n}
$$

i.e., on the open set where the traces of the powers of the Nijenhuis tensor are functionally independent, we have that the eigenvalues $\lambda_{i}$ are different and functionally independent.

To proceed further we need to recall [33] that the normalized traces $I_{k}$ of the powers of Nijenhuis operator satisfy the recursion relation

$$
N^{*} d I_{k}=d I_{k+1} .
$$

This can be proved as follows. At first one notices that (2.11) is equivalent to the relation

$$
L_{N X}\left(I_{k}\right)=L_{X}\left(I_{k+1}\right) \quad \text { for all vector field } X,
$$

as it can be easily seen evaluating the equality (2.11) on a generic vector field $X$. Thanks to the Leibniz property of the Lie derivative and the cyclicity of the trace, we see that

$$
L_{N X}\left(I_{k}\right)=\operatorname{Tr}\left(L_{N X}(N) \cdot N^{k-1}\right) \quad \text { and } L_{X}\left(I_{k+1}\right)=\operatorname{Tr}\left(L_{X}(N) \cdot N^{k}\right) .
$$

Since the vanishing of the Nijenhuis torsion of $N$ implies that $L_{N X}(N)=N \cdot L_{X}(N)$, the validity of $(2.11)$ is proved.

We now express the relations (2.11) in terms of the eigenvalues $\lambda_{i}$ as

$$
\left[\begin{array}{cccc}
1 & 1 & \cdots & 1 \\
\lambda_{1} & \lambda_{2} & \cdots & \lambda_{n} \\
\vdots & \vdots & \ddots & \vdots \\
\lambda_{1}^{n-1} & \lambda_{2}^{n-1} & \cdots & \lambda_{n}^{n-1}
\end{array}\right] \cdot\left[\begin{array}{c}
N^{*} d \lambda_{1}-\lambda_{1} d \lambda_{1} \\
N^{*} d \lambda_{2}-\lambda_{2} d \lambda_{2} \\
\vdots \\
N^{*} d \lambda_{n}-\lambda_{n} d \lambda_{n}
\end{array}\right]=\left[\begin{array}{c}
0 \\
0 \\
\vdots \\
0
\end{array}\right]
$$


Since the Vandermond matrix in the left-hand side of this equation is, by assumption, invertible, we conclude that $N^{*} d \lambda_{i}=\lambda_{i} d \lambda_{i}$ for all $i=1, \ldots, n$.

This proposition can be rephrased saying that "half of" the DN coordinates are algebraically provided by the Nijenhuis tensor itself. The remaining "half" $\mu_{1}, \ldots, \mu_{n}$ can always be found by quadratures. Actually, there is a condition leading to the algebraic solution of this problem too. To elucidate this, the following two considerations are crucial.

The first argument goes as follows. Let us consider the distinguished functions $I_{k}$ introduced in Proposition 2.9, and trade them for the coefficients $p_{i}$ of the minimal polynomial

$$
\Delta_{N}(\lambda)=\lambda^{n}-p_{1} \lambda^{n-1}-p_{2} \lambda^{n-2}-\cdots-p_{n}
$$

of $N$. The functions $p_{k}$ and $I_{k}$ are related by the triangular Newton formulas

$$
\begin{aligned}
& I_{1}=p_{1} ; \quad I_{2}=p_{2}+\frac{1}{2} p_{1}^{2} ; \quad I_{3}=p_{3}+p_{2} p_{1}+\frac{1}{3} p_{1}^{3} \\
& I_{4}=p_{4}+p_{1} p_{3}+p_{1}^{2} p_{2}+\frac{1}{2} p_{2}^{2}+\frac{1}{4} p_{1}^{4} ; \quad I_{5}=p_{5}+\ldots
\end{aligned}
$$

As a consequence of the recursion relations (2.11), it can be easily shown that the $p_{i}$ 's satisfy the 'Frobenius' recursion relations

$$
N^{*} d p_{i}=d p_{i+1}+p_{i} d p_{1} \text {, with } p_{n+1} \equiv 0 .
$$

We can compactly write these relations as a single relation for the polynomial $\Delta_{N}(\lambda)$; indeed, a straightforward computation shows that they are equivalent to

$$
N^{*} d \Delta_{N}(\lambda)=\lambda d \Delta_{N}(\lambda)+\Delta_{N}(\lambda) d p_{1}
$$

Actually, relations of this kind are very important for our purposes. Indeed, in [21] we proved the following

Proposition 2.10 Let $\Phi(\lambda)$ be a smooth function defined on the manifold $M$, depending on an additional parameter $\lambda$. Suppose that there exists a one-form $\alpha_{\Phi}$ such that

$$
N^{*} d \Phi(\lambda)=\lambda d \Phi(\lambda)+\Delta_{N}(\lambda) \alpha_{\Phi} .
$$

Then, the $n$ functions $\Phi_{i}$ obtained evaluating the "generating" function $\Phi(\lambda)$ for $\lambda=\lambda_{i}$, with $i=1, \ldots, n$, are Nijenhuis functions, that is, they satisfy $N^{*} d \Phi_{i}=\lambda_{i} d \Phi_{i}$.

Definition 2.11 We will call a generating function $\Phi(\lambda)$ satisfying equation (2.17) a Nijenhuis functions generator. 
Secondly, one remarks [21] that the $n(n-1) / 2$ equations $\left\{\lambda_{i}, \mu_{j}\right\}=\delta_{i j}$ can be replaced with the requirement $N^{*} d \mu_{j}=\lambda_{j} d \mu_{j}$ and the $n$ equations

$$
\left\{\lambda_{1}+\cdots+\lambda_{n}, \mu_{j}\right\}=1, \quad j=1, \ldots, n,
$$

that do not involve the individual coordinates $\lambda_{i}$ but only their sum $\sum_{i=1}^{n} \lambda_{i}=I_{1}$. In terms of the Hamiltonian vector field $Y=-P d I_{1}=\sum_{i} \frac{\partial}{\partial \mu_{i}}$, the condition we are looking for is

$$
L_{Y}\left(\mu_{i}\right)=1
$$

The relevance of Definition 2.11 in the search for DN coordinates stems from the fact that Nijenhuis functions generators form an algebra $\mathcal{N}(M)$, which is closed under the action of the vector field $Y=-P d I_{1}$. In this way, knowing a set of Nijenhuis functions generators, we can obtain further elements of the algebra $\mathcal{N}(M)$ by repeated applications of the vector field $Y$. Clearly, in such an extended algebra, the characteristic equation

$$
L_{Y}(\Psi(\lambda))=1+\Delta_{N}(\lambda) f_{\Psi}
$$

corresponding to (2.18), may be easier to solve, thus yielding the missing DarbouxNijenhuis coordinates $\mu_{i}$ as $\mu_{i}=\Psi\left(\lambda_{i}\right)$. The following remark is very important in view of the relations with algebraic integrability.

Remark 2.12 Suppose $\left(H_{1}, \ldots, H_{n}\right)$ to be a separable system in the DN coordinates constructed above. Then the separation relations (1.3) do not depend on the pair $\left(\lambda_{i}, \mu_{i}\right)$, i.e., they collapse to the single relation

$$
\Phi\left(\lambda, \mu ; H_{1}, \ldots, H_{n}\right)=0 .
$$

Indeed, $\mu_{i}=\Psi\left(\lambda_{i}\right)$, where the $\lambda_{i}$ are the eigenvalues of $N$. Since the $I_{k}$ are invariant with respect to the exchange $\lambda_{i} \leftrightarrow \lambda_{j}$, every function globally defined on $M$ is invariant with respect to the exchange $\left(\lambda_{i}, \mu_{i}\right) \leftrightarrow\left(\lambda_{j}, \mu_{j}\right)$. This is in particular true for the Hamiltonians $H_{k}$, and the assertion about (2.19) follows.

In many cases, equation (2.19) defines an algebraic curve, possibly coinciding with the spectral curve associated with a Lax matrix for the Hamiltonian system at hand. We will see an instance of this situation in the example of Section 5. The application of this scheme to (a particular class of) Gaudin models have been spelled out in [19, 19]

\section{Separability conditions in the bihamiltonian set- ting}

As we have briefly recalled in Section 2, on a bihamiltonian manifold one is usually led to consider bihamiltonian vector fields, that is, vector fields $X$ admitting the twofold Hamiltonian representation $X=P d f=P^{\prime} d g$. Let us now suppose that $\left(M, P, P^{\prime}\right)$ be 
a regular bihamiltonian manifold of dimension $2 n$, and that we were able to construct, by means of the Lenard-Magri iteration procedure, a sequence of functions $H_{1}, H_{2}, \ldots$ satisfying $P^{\prime} d H_{i}=P d H_{i+1}$. Let us also suppose that the first $n$ of them be functionally independent. Then one easily shows that all the further Hamiltonians $H_{n+1}, \ldots$ are functionally dependent from the first $n$. (This follows from the fact that a regular Poisson manifold of dimension $2 n$ cannot have more than $n$ mutually commuting independent functions). This means that, if we consider the Hamiltonian $H_{n+1}$, there must be a relation of the form

$$
\psi\left(H_{1}, \ldots, H_{n} ; H_{n+1}\right)=0, \quad \text { with } \psi_{H_{n+1}} \equiv \frac{\partial \psi}{\partial H_{n+1}} \neq 0,
$$

relating it with the independent Hamiltonians $H_{i}$, with $i=1, \ldots, n$.

Actually, the case of $H_{i}=I_{i} \equiv \frac{1}{2 i} \operatorname{Tr} N^{i}$ is an instance of this situation. In fact, since by the Cayley-Hamilton theorem $N$ annihilates its minimal polynomial, we have $N^{n}-\sum_{i=1}^{n} p_{i} N^{n-i}=0$, yielding the relation

$$
2(n+1) I_{n+1}-\sum_{i=1}^{n} 2(n-i+1) p_{i} I_{n-i+1}=0 .
$$

Differentiating equation (3.1) we see that, along with $P^{\prime} d H_{i}=P d H_{i+1}$, for $i=1, \ldots, n-$ 1, it holds:

$$
P^{\prime} d H_{n}=P d H_{n+1}=-\frac{1}{\psi_{H_{n+1}}} \sum_{i=1}^{n} \frac{\partial \psi}{\partial H_{i}} P d H_{i},
$$

that is, the vector field $X_{n+1}=P d H_{n+1}=P^{\prime} d H_{n}$ is a linear combination of the vector fields $X_{1}=P d H_{1}, \ldots, X_{n}=P d H_{n}$.

This innocent looking observation is the clue for the bihamiltonian theory of SoV. Indeed, let $\left\{H_{1}, H_{2}, \ldots H_{n}\right\}$ be any integrable system on $M$, that is, suppose that the $H_{i}$ are mutually commuting (with respect to $P$ ) independent functions. We can construct an $n$-dimensional distribution, namely the distribution $\mathcal{D}_{H}$ spanned by the $n$ mutually commuting vector fields $X_{i}=P d H_{i}$. This is nothing but the very classical tangent distribution to the invariant (generalized) tori of the Liouville Arnol'd theory of integrable systems. Since $M$ comes equipped with a second Poisson tensor $P^{\prime}$, we can as well consider the distribution $\mathcal{D}_{H}^{\prime}$ generated by the Hamiltonians $H_{i}$ under the action of $P^{\prime}$, that is, generated by the vector fields $X_{i}^{\prime}=P^{\prime} d H_{i}$. It holds:

Theorem 3.1 Let $\left\{H_{1}, \ldots, H_{n}\right\}$ define, as explained above, an integrable system on a regular bihamiltonian manifold $\left(M, P, P^{\prime}\right)$. The Hamilton-Jacobi equations associated with any of the Hamiltonians $H_{i}$ are separable in the $D N$ coordinates $\left(x_{1}, \ldots, x_{n}, y_{1}, \ldots, y_{n}\right)$ defined by $N=P^{\prime} P^{-1}$ if and only if the distribution $\mathcal{D}_{H}^{\prime}$ is contained in $\mathcal{D}_{H}$, or, equivalently, if and only if the distribution $\mathcal{D}_{H}$ is invariant along $N$.

Proof. We will first prove the equivalence between the invariance of $\mathcal{D}_{H}$ under $N$ and the inclusion $\mathcal{D}_{H}^{\prime} \subset \mathcal{D}_{H}$. To say that $\mathcal{D}_{H}^{\prime}$ is contained in $\mathcal{D}_{H}$ is tantamount to saying 
that there exists a matrix $F_{i j}$, whose entries are, in general, functions defined on $M$, such that

$$
X_{i}^{\prime} \equiv P^{\prime} d H_{i}=\sum_{j} F_{i j} P d H_{j}=\sum_{j} F_{i j} X_{j} \quad \text { for } i=1, \ldots, n .
$$

Writing $P^{\prime}=N P$, we can translate these equalities into $N X_{i}=\sum_{j} F_{i j} X_{j}$ for all $i=$ $1, \ldots, n$.

The full proof of the fact that the invariance of $\mathcal{D}_{H}$ insures separability in DN coordinates can be found in [21]. It goes as follows.

At first we notice that the translation in terms of the codistribution $\mathcal{D}_{H}^{*}$ generated by the differentials of the Hamiltonians $H_{i}$ of the invariance condition for $\mathcal{D}_{H}$ is the invariance condition $N^{*} \mathcal{D}_{H}^{*} \subset \mathcal{D}_{H}^{*}$. This can be easily seen applying to (3.3) the operator $P^{-1}$, to get $N^{*} d H_{i}=\sum_{j} F_{i j} d H_{j}$.

Since all the Poisson brackets $\left\{H_{i}, H_{j}\right\}$ vanish and $M$ is a regular bihamiltonian manifold, the matrix $F$ defined by (3.3) can be shown to have simple eigenvalues, that coincide with the eigenvalues $\lambda_{i}$ of $N$. So there exists a matrix $S$ satisfying $S F=\Lambda S$, where $\Lambda=\operatorname{diag}\left(\lambda_{1}, \ldots, \lambda_{n}\right)$ If we introduce the $n$ one-forms $\theta_{i}=\sum_{j} S_{i j} d H_{j}$, we get

$$
N^{*} \theta_{i}=\sum_{j} S_{i j} N^{*} d H_{j}=\sum_{j, k} S_{i j} F_{j k} d H_{k}=\sum_{j, k} \lambda_{i} \delta_{i j} S_{j k} d H_{k}=\lambda_{i} \theta_{i},
$$

meaning that $\theta_{i}$ is an eigenvector of $N^{*}$ relative to $\lambda_{i}$. Hence there must exist functions $F_{i}, G_{i}$ such that

$$
\sum_{j} S_{i j} d H_{j}=F_{i} d x_{i}+G_{i} d y_{i}
$$

whence the existence of a separation relation $\Phi_{i}\left(x_{i}, y_{i} ; H_{1}, \ldots, H_{n}\right)$ for all $i=1, \ldots, n$. The converse statement can be trivially proved.

We would like to stress that the separability condition of Theorem 3.1 is a tensorial one. That is, given a regular bihamiltonian manifold $\left(M, P, P^{\prime}\right)$ this separability criterion can be checked in any system of coordinates, without the a priori calculation of the DN coordinates themselves. Notice, also, that the validity of the statement does not (as it should be!) depend on the choice of mutually commuting integrals $\left\{H_{1}, \ldots, H_{n}\right\}$. That is, if we consider a "change of coordinates in the space of the actions", that is, we trade the $H_{i}$ 's for another complete set of integrals of the motion $K_{i}=K_{i}\left(H_{1}, \ldots, H_{n}\right)$, then the separability of the new Hamiltonians $K_{i}$ will hold if and only if the separability of the original ones holds. Indeed, the dual distributions generated by the $H_{i}$ 's and the $K_{i}$ 's coincide.

A second remark is important and deserves to be explicitly spelled out. Although we have started our discussion considering the case of a family of bihamiltonian vector fields, that is, the case of Lenard-Magri sequences, the hypotheses of Theorem 3.1 concern only 
the relations of the distributions generated respectively under the action of $P$ and $P^{\prime}$ by the Hamiltonians $H_{i}$, without any mention of the fact that the generators of the distribution be bihamiltonian vector fields. Thus, although it might seem a somewhat odd statement, the vector fields that are separable by means of the bihamiltonian approach are not necessarily bihamiltonian vector fields! It is also important to notice that it is not only a matter of choice of generators. Indeed, in [35] it has been shown that the only bihamiltonian vector fields on a regular bihamiltonian manifold turn out to be associated with separated functions of the eigenvalues of $N$, i.e., functions of the form $H=\sum_{i=1}^{n} f_{i}\left(\lambda_{i}\right)$. This means that, in such a case, the distribution $\mathcal{D}_{H}$ coincides with that generated by the distinguished functions $I_{i}$. However, this is by far a very special example, that is, the range of applicability of the method is much wider than that, as it has already been shown in the literature.

The separation condition of Theorem 3.1 is based on the analysis of the behaviour of the characteristic distribution associated with an integrable system under the Nijenhuis tensor $N$. An equivalent criterion, based on the analysis of the Poisson brackets associated with the tensor $P^{\prime}$, can be formulated as follows.

Theorem 3.2 Let $\left\{H_{1}, H_{2}, \ldots H_{n}\right\}$ be an integrable system defined on a regular bihamiltonian manifold $\left(M, P, P^{\prime}\right)$. The Hamiltonians $H_{i}$ are separable in the $D N$ coordinates defined by $N=P^{\prime} P^{-1}$ if and only if, along with the commutation relations $\left\{H_{i}, H_{j}\right\}=0$, there also hold

$$
\left\{H_{i}, H_{j}\right\}^{\prime} \equiv\left\langle d H_{i}, P^{\prime} d H_{j}\right\rangle=0, \text { for } i, j=1, \ldots, n .
$$

Proof. The key formula is the relation between $P, P^{\prime}$ and $N^{*}$. Indeed, suppose that $\mathcal{D}_{H}^{*}$ be invariant along $N^{*}$. Then:

$$
\begin{aligned}
\left\{H_{i}, H_{j}\right\}^{\prime} & =\left\langle d H_{i}, P^{\prime} d H_{j}\right\rangle=\left\langle d H_{i}, N P d H_{j}\right\rangle=\left\langle N^{*} d H_{i}, P d H_{j}\right\rangle \\
& =\sum_{k} F_{i k}\left\langle d H_{k}, P d H_{j}\right\rangle=\sum_{k} F_{i k}\left\{H_{k}, H_{i}\right\}=0,
\end{aligned}
$$

which, in view of Theorem 3.1, proves the statement in one direction. Now, let us suppose that (3.6) holds. Then, for every $i, j=1, \ldots, n$, we have:

$$
0=\left\{H_{i}, H_{j}\right\}^{\prime}=\left\langle d H_{i}, P^{\prime} d H_{j}\right\rangle=\left\langle d H_{i}, N P d H_{j}\right\rangle=\left\langle N^{*} d H_{i}, P d H_{j}\right\rangle,
$$

meaning that, for all $i=1, \ldots, n$, the one-form $N^{*} d H_{i}$ belongs to the annihilator (with respect to $P$ ) of the distribution $\mathcal{D}_{H}$. Since such an annihilator coincides with $\mathcal{D}_{H}^{*}$, this means that $N^{*} d H_{i} \in \mathcal{D}_{H}^{*}$ for all $i=1, \ldots, n$.

This results lead to the following, (somewhat daring), comparison. The Liouville-Arnol'd theorem on finite dimensional integrable Hamiltonian systems says that the geometrical 
structure underlying integrability of a Hamiltonian vector field defined on a symplectic manifold $(M, \omega)$ is a Lagrangian foliation of $M$. We can rephrase the content of Theorem 3.2 saying that the geometrical structure underlying the separability of a system defined on a regular bihamiltonian manifold $\left(M, P, P^{\prime}\right)$ is a bilagrangian foliation of $M$.

We end our presentation of the bihamiltonian set-up for SoV with the following remark. Theorem 3.1 concerns only the existence of the separation relations. In principle, one could try to find these relations in concrete examples by actually diagonalizing the matrix $F$, and explicitly finding and integrating the relations (3.5). However, there is a very simple tensorial criterion which can be used to determine the functional form of the separation relations $\Phi_{i}\left(x_{i}, y_{i} ; H_{1}, \ldots, H_{n}\right)$, whose proof can be found in [21].

Proposition 3.3 Let $\left\{H_{1}, \ldots, H_{n}\right\}$ be an integrable system defined on a regular bihamiltonian manifold, which is separable in the Darboux-Nijenhuis coordinates associated with $N=P^{-1} P^{\prime}$. Consider the matrix $F_{i j}$ fulfilling the relations (3.3). Then the separation relations are affine in the Hamiltonians $H_{i}$, that is, of the form

$$
\Phi_{i}\left(x_{i}, y_{i} ; H_{1}, \ldots, H_{n}\right)=\sum_{j} S_{i j}\left(x_{i}, y_{i}\right) H_{j}+U_{i}\left(x_{i}, y_{i}\right),
$$

if and only if the matrix $F$ satisfies the relation $N^{*} d F_{i j}=\sum_{k} F_{i k} d F_{k j}$.

The matrix $S$ on (3.7) can be shown to be a suitably normalized matrix of eigenvectors of the matrix $F$. Its characteristic property is that, as expressed in the equation, the entries $S_{i j}$ of the $i$-th row depend only on the pair $\left(x_{i}, y_{i}\right)$ of Darboux-Nijenhuis coordinates. For this reason it can be called a Stäckel matrix.

\section{Transversal distributions and separation relations}

A very natural source of integrable systems fulfilling the separability conditions given in Theorems 3.1 and 3.2 is described in [21]. In this short section we recall this construction, and we comment on the resulting separation relations, with a particular emphasis on the relations with algebraic integrability.

Suppose that the hypotheses of Proposition 2.7 hold, and that there exists a $k$ dimensional foliation $\mathcal{Z}$ on $M$, spanned by the vector fields $Z_{1}, \ldots, Z_{k}$, with the following properties:

1. It is transversal to the symplectic foliation of $P$; more precisely, the vector fields $Z_{a}$ are normalized in such a way that $Z_{a}\left(H_{0}^{(b)}\right)=\delta_{a}^{b}$;

2. The $Z_{a}$ are symmetries of $P$ :

$$
L_{Z_{a}}(P)=0
$$

3. There exist vector fields $Y_{a}^{b}$ such that

$$
L_{Z_{a}} P^{\prime}=\sum_{b} Y_{a}^{b} \wedge Z_{b}
$$


It turns out that the $Z_{a}$ commute, and that $Y_{a}^{b}=P d\left(Z_{a}\left(H_{1}^{(b)}\right)\right)$. But the important point is that any symplectic leaf $\mathcal{S}$ of $P$ can be seen as a quotient space and inherits a (quotient) bi-Hamiltonian structure from $M$. Moreover, the reduction of $P$ coincides with the symplectic form of $\mathcal{S}$, and therefore $\mathcal{S}$ is a regular bihamiltonian manifold (if the eigenvalues of the associated Nijenhuis tensor are maximally distinct).

Now, it can be shown that the integrable system described in Proposition 2.7 is separable in the DN coordinates on $\mathcal{S}$. As far as the Stäckel separability is concerned, a necessary and sufficient condition is that $Z_{b}\left(Z_{c}\left(H_{j}^{(a)}\right)\right)=0$ on $\mathcal{S}$, for all $a, b, c=1, \ldots, k$ and for all $j=1, \ldots, n_{a}$.

The search for DN coordinates is made easier by the fact that the determinant of the matrix

$$
\mathcal{G}(\lambda)=\left(\begin{array}{ccc}
Z_{1}\left(H^{(1)}(\lambda)\right) & \cdots & Z_{k}\left(H^{(1)}(\lambda)\right) \\
\vdots & & \vdots \\
Z_{1}\left(H^{(k)}(\lambda)\right) & \cdots & Z_{k}\left(H^{(k)}(\lambda)\right)
\end{array}\right)
$$

coincides on $\mathcal{S}$ with the minimal polynomial of the recursion operator $N$. Thus the coordinates $\lambda_{i}$ are the solutions of $\mathcal{G}(\lambda)=0$. To find the $\mu_{i}$, one can use the results of Subsection 2.2 and the following proposition, whose proof is given in [22].

Proposition 4.1 In the above setting, let us consider a generating function $\Gamma(\lambda, \mu)$ of the Casimirs $H^{(a)}(\lambda)$ of the Poisson pencil, and let us suppose that $\Gamma(\lambda, \mu)=0$ defines a smooth algebraic curve. Suppose that $f$ is a $\mathcal{Z}$-invariant root of the minimal polynomial of $N$, i.e.,

$$
N^{*} d f=f d f, \quad \text { and } Z_{i}(f)=0, i=1, \ldots, k,
$$

and suppose that $\Gamma(\mu, f)=0$ defines generic point(s) of the affine curve $\Gamma(\lambda, \mu)=0$. Then, any solution $g$ of the equation $\Gamma(g, f)=0$ which is invariant as well under $\mathcal{Z}$ satisfies $N^{*} d g=f d g$.

\section{Example: a generalized Toda Lattice}

In this final section we will apply the general scheme outlined in the previous sections to a specific model, with the aim of showing how the recipes discussed so far from a theoretical standpoint can be concretely applied. We will study a generalization of the four site Toda lattice, to be termed Toda $a_{3}^{4}$ model. This system is a member of a family introduced in [28] as reductions of the discrete KP hierarchy. It can be described as follows. We consider on $M=\mathbb{C}^{12}$, endowed with global coordinates $\left\{b_{i}, a_{i}, c_{i}\right\}_{i=1,2,3,4}$, the Hamiltonian

$$
H_{G T}=\frac{1}{2}\left(b_{1}^{2}+b_{2}^{2}+b_{3}^{2}+b_{4}^{2}\right)-\left(a_{1}+a_{2}+a_{3}+a_{4}\right),
$$


and the linear Poisson tensor given by the matrix

$$
\begin{aligned}
& P=\left[\begin{array}{ccc}
\mathbf{0} & \mathbf{A}_{\mathbf{1}} & \mathbf{C}_{\mathbf{1}} \\
-\mathbf{A}_{\mathbf{1}}^{\mathbf{T}} & \mathbf{C}_{\mathbf{2}} & \mathbf{0} \\
-\mathbf{C}_{\mathbf{1}}^{\mathbf{T}} & \mathbf{0} & \mathbf{0}
\end{array}\right] \text { where } \mathbf{A}_{\mathbf{1}}=\left[\begin{array}{cccc}
-a_{1} & 0 & 0 & a_{4} \\
a_{1} & -a_{2} & 0 & 0 \\
0 & a_{2} & -a_{3} & 0 \\
0 & 0 & a_{3} & -a_{4}
\end{array}\right] \\
& \mathbf{C}_{\mathbf{1}}=\left[\begin{array}{cccc}
-c_{1} & 0 & c_{3} & 0 \\
0 & -c_{2} & 0 & c_{4} \\
c_{1} & 0 & -c_{3} & 0 \\
0 & c_{2} & 0 & -c_{4}
\end{array}\right], \quad \mathbf{C}_{\mathbf{2}}=\left[\begin{array}{cccc}
0 & -c_{1} & 0 & c_{4} \\
c_{1} & 0 & -c_{2} & 0 \\
0 & c_{2} & 0 & -c_{3} \\
-c_{4} & 0 & c_{3} & 0
\end{array}\right]
\end{aligned}
$$

and we denoted by $\mathbf{0}$ the $4 \times 4$ matrix with vanishing entries. Using (here and in the sequel) the cyclic identifications $a_{i+4}=a_{i}, b_{i+4}=b_{i}$, and $c_{i+4}=c_{i}$, the Hamiltonian vector field $X_{H_{G T}}=P d H_{G T}$ can be written as

$$
\left[\begin{array}{c}
\dot{b}_{i} \\
\dot{a}_{i} \\
\dot{c}_{i}
\end{array}\right]=\left[\begin{array}{l}
a_{i-1}-a_{i} \\
a_{i}\left(b_{i+1}-b_{i}\right)+c_{i-1}-c_{i} \\
c_{i}\left(b_{i-2}-b_{i}\right)
\end{array}\right], \quad i=1, \ldots, 4 .
$$

The expert reader surely noticed that $H_{G T}$ coincides with the Hamiltonian of the periodic four-site Toda lattice, written in the Flaschka coordinates $b_{i}=p_{i}, a_{i}=\exp \left(q_{i}-q_{i+1}\right)$. Indeed, on the hyperplane $M_{T} \simeq \mathbb{C}^{8}$ defined by $c_{i}=0$ for $i=1, \ldots, 4$, the vector field $X_{H_{G T}}$ defines the periodic Toda flow.

Proposition 5.1 The Hamiltonian vector field $X_{H_{G T}}$ admits the Lax representation $\dot{L}(\nu)=[L(\nu), \Phi]$, where

$$
L(\nu)=\left[\begin{array}{cccc}
b_{1} & -\nu & \frac{c_{3}}{\nu^{2}} & \frac{a_{4}}{\nu} \\
\frac{a_{1}}{\nu} & b_{2} & -\nu & \frac{c_{4}}{\nu^{2}} \\
\frac{c_{1}}{\nu^{2}} & \frac{a_{2}}{\nu} & b_{3} & -\nu \\
-\nu & \frac{c_{2}}{\nu^{2}} & \frac{a_{3}}{\nu} & b_{4}
\end{array}\right], \quad \Phi=\left[\begin{array}{cccc}
0 & 0 & \frac{c_{3}}{\nu^{2}} & \frac{a_{4}}{\nu} \\
\frac{a_{1}}{\nu} & 0 & 0 & \frac{c_{4}}{\nu^{2}} \\
\frac{c_{1}}{\nu^{2}} & \frac{a_{2}}{\nu} & 0 & 0 \\
0 & \frac{c_{2}}{\nu^{2}} & \frac{a_{3}}{\nu} & 0
\end{array}\right]
$$

The bihamiltonian aspects of this system have been discussed in [36] (see also [10]). In particular, it has been noticed that on $M$ there exists a second Hamiltonian structure for the vector field $X_{H_{G T}}$. Namely, one considers the bivector $P^{\prime}$ having the following form:

$$
P^{\prime}=\left[\begin{array}{ccc}
\mathbf{A}_{\mathbf{2}} & \mathbf{B}_{\mathbf{1}} & \mathbf{C}_{\mathbf{3}} \\
-\mathbf{B}_{1}^{\mathbf{T}} & \mathbf{A}_{\mathbf{3}} & \mathbf{C}_{\mathbf{4}} \\
-\mathbf{C}_{\mathbf{3}}^{\mathbf{T}} & -\mathbf{C}_{\mathbf{4}}^{\mathbf{T}} & \mathbf{A}_{\mathbf{4}}
\end{array}\right] \text {, where } \mathbf{C}_{\mathbf{3}}=\left[\begin{array}{cccc}
-b_{1} c_{1} & 0 & b_{1} c_{3} & 0 \\
0 & -b_{2} c_{2} & 0 & b_{2} c_{4} \\
c_{1} b_{3} & 0 & -b_{3} c_{3} & 0 \\
0 & c_{2} b_{4} & 0 & -b_{4} c_{4}
\end{array}\right]
$$




$$
\begin{aligned}
& \mathbf{A}_{2}=\left[\begin{array}{cccc}
0 & a_{1} & 0 & -a_{4} \\
-a_{1} & 0 & a_{2} & 0 \\
0 & -a_{2} & 0 & a_{3} \\
a_{4} & 0 & -a_{3} & 0
\end{array}\right], \quad \mathbf{B}_{1}=\left[\begin{array}{cccc}
-b_{1} a_{1} & c_{1} & -c_{3} & b_{1} a_{4} \\
b_{2} a_{1} & -b_{2} a_{2} & c_{2} & -c_{4} \\
-c_{1} & b_{3} a_{2} & -b_{3} a_{3} & c_{3} \\
c_{4} & -c_{2} & b_{4} a_{3} & -b_{4} a_{4}
\end{array}\right] \\
& \mathbf{A}_{3}=\left[\begin{array}{cccc}
0 & -b_{2} c_{1}-a_{1} a_{2} & 0 & b_{1} c_{4}+a_{1} a_{4} \\
b_{2} c_{1}+a_{1} a_{2} & 0 & -b_{3} c_{2}-a_{2} a_{3} & 0 \\
0 & b_{3} c_{2}+a_{2} a_{3} & 0 & -b_{4} c_{3}-a_{3} a_{4} \\
-b_{1} c_{4}-a_{1} a_{4} & 0 & b_{4} c_{3}+a_{3} a_{4} & 0
\end{array}\right] \text {, } \\
& \mathbf{C}_{4}=\left[\begin{array}{cccc}
-a_{1} c_{1} & -a_{1} c_{2} & a_{1} c_{3} & a_{1} c_{4} \\
c_{1} a_{2} & -a_{2} c_{2} & -a_{2} c_{3} & a_{2} c_{4} \\
c_{1} a_{3} & c_{2} a_{3} & -a_{3} c_{3} & -a_{3} c_{4} \\
-c_{1} a_{4} & c_{2} a_{4} & c_{3} a_{4} & -a_{4} c_{4}
\end{array}\right], \quad \mathbf{A}_{4}=\left[\begin{array}{cccc}
0 & -c_{1} c_{2} & 0 & c_{1} c_{4} \\
c_{1} c_{2} & 0 & -c_{2} c_{3} & 0 \\
0 & c_{2} c_{3} & 0 & -c_{3} c_{4} \\
-c_{1} c_{4} & 0 & c_{3} c_{4} & 0
\end{array}\right] .
\end{aligned}
$$

It can be easily checked that $X_{H_{G T}}=P^{\prime} d\left(-\sum_{i=1}^{4} b_{i}\right)$. More in general, we have the following

Proposition 5.2 The pencil $P^{\prime}-\lambda P$ is a pencil of Poisson brackets. The rank of the generic element of the pencil is eight. The characteristic polynomial $\operatorname{Det}(\lambda \mathbf{1}-L(\nu))$ can be expanded as:

$$
\operatorname{Det}(\lambda \mathbf{1}-L(\nu))=\lambda^{4}-\nu^{4}+H(\lambda)+\left(K(\lambda)-\lambda^{2} J_{1}\right) / \nu^{4}+J_{2} / \nu^{8} .
$$

The functions $J_{1}$ and $J_{2}$ are common Casimirs of $P$ and $P^{\prime}$. The polynomials $H(\lambda)$ and $K(\lambda)$ are Casimirs of the pencil $P_{\lambda}=P^{\prime}-\lambda P$. They have the form

$$
H(\lambda)=\lambda^{3} H_{0}-\lambda^{2} H_{1}+\lambda H_{2}-H_{3}, \quad K(\lambda)=K_{0} \lambda+K_{1} .
$$

Explicitly, $J_{1}=c_{1} c_{3}+c_{2} c_{4}$ and $J_{2}=c_{1} c_{2} c_{3} c_{4}$, while the coefficients of $H(\lambda)$ and $K(\lambda)$ are given by:

$$
\begin{aligned}
& H_{0}=\sum_{i=1}^{4} b_{i}, H_{1}=\sum_{i>j=1}^{4} b_{i} b_{j}+\sum_{i=1}^{4} a_{i}, H_{2}=\sum_{i=1}^{4}\left(c_{i}+b_{i}\left(a_{i+1}+a_{1+2}\right)+b_{i} b_{i+1} b_{i+2}\right) \\
& H_{3}=\sum_{i=1}^{4} b_{i} c_{i+1}+a_{1} a_{3}+a_{2} a_{4}+\text { cubic and quartic terms; } \\
& K_{0}=\sum_{i=1}^{4}\left(b_{i} c_{i-1} c_{i+1}-c_{i} a_{i-1} a_{i+2}\right), K_{1}=\sum_{i=1}^{4} a_{i} c_{i+1} c_{i+2}+\text { quartic terms }
\end{aligned}
$$

One can show via a direct computation that the eight functions $H_{0}, H_{1}, H_{2}, H_{3}, K_{0}, K_{1}, J_{1}, J_{2}$ are functionally independent and, thanks to the fact that they fill in Lenard sequences, 
are mutually in involution. The kernel of $P$ is generated (at generic points $m \in M$ ) by the differentials of the four functions $H_{0}, K_{0}, J_{1}, J_{2}$. Hence, on the 8-dimensional manifold $\mathcal{S}_{\kappa}$ defined by the equations $H_{0}=\kappa_{1}, K_{0}=\kappa_{2}, J_{1}=\kappa_{3}, J_{2}=\kappa_{4}$, that is, the generic symplectic leaf of $P$, the vector field $X_{H_{G T}}$ is completely integrable. To realize this we simply have to notice that $H_{G T}$ can be expressed as $\frac{1}{2} H_{0}^{2}-H_{1}$, and apply the properties of anchored Lenard-Magri sequences collected in Proposition 2.7

\subsection{Separation of Variables}

We will now show how to apply the ideas and recipes of the bihamiltonian set-up for SoV to the Toda ${ }_{3}^{4}$ model introduced above. The first problem to deal with is that the Poisson tensor $P^{\prime}$ does not restrict to $\mathcal{S}_{\boldsymbol{\kappa}}$, but must be projected according to the procedure outlined in Section 4. This can be rephrased as follows, by means of a kind of Dirac reduction process (see [11, 20, 21, 34] for details and the geometric background). matrix

We consider the vector fields $Z_{1}=-\frac{\partial}{\partial b_{4}}$ and $Z_{2}=\frac{\partial}{\partial a_{4}}$, and we notice that the

$$
G=\left(\begin{array}{ll}
L_{Z_{1}}\left(H_{0}\right) & L_{Z_{2}}\left(H_{0}\right) \\
L_{Z_{1}}\left(K_{0}\right) & L_{Z_{2}}\left(K_{0}\right)
\end{array}\right)=\left(\begin{array}{cc}
1 & 0 \\
-c_{1} c_{3} & -c_{1} a_{3}-a_{1} c_{2}
\end{array}\right)
$$

is invertible. Then we form the bivector

$$
R=\sum_{i, j=1}^{2}\left(G^{-1}\right)_{i j} Z_{i} \wedge X_{1}^{j}, \text { where } X_{1}^{1}=P^{\prime} d H_{0} \text { and } X_{1}^{2}=P^{\prime} d K_{0} .
$$

Lemma 5.3 The modified bivector $Q=P^{\prime}-R$ defines a Poisson bracket, compatible with $P$; moreover, $Q$ restricts to $\mathcal{S}_{\kappa}$.

Proof. The proof of the fact that $Q_{\lambda}=Q-\lambda P$ is a Poisson pencil follows (see, e.g., $[20])$, from the equalities

$$
\begin{gathered}
L_{Z_{1}} P=0, \quad L_{Z_{1}} P^{\prime}=Y_{1}^{1} \wedge Z_{1}-c_{3} \frac{\partial}{\partial a_{3}} \wedge Z_{2} \\
L_{Z_{2}} P=\left(\frac{\partial}{\partial b_{1}}-\frac{\partial}{\partial a_{1}}\right) \wedge Z_{2}, \quad L_{Z_{2}} P^{\prime}=\left(b_{4} \frac{\partial}{\partial a_{4}}+\frac{\partial}{\partial b_{1}}\right) \wedge Z_{1}+Y_{2}^{2} \wedge Z_{2},
\end{gathered}
$$

where

$$
\begin{aligned}
& Y_{1}^{1}=a_{3} \frac{\partial}{\partial a_{3}}-a_{4} \frac{\partial}{\partial a_{4}}+c_{2} \frac{\partial}{\partial c_{2}}-c_{4} \frac{\partial}{\partial c_{4}} \\
& Y_{2}^{2}=b_{4} \frac{\partial}{\partial b_{4}}-b_{1} \frac{\partial}{\partial b_{1}}-a_{3} \frac{\partial}{\partial a_{3}}-a_{4} \frac{\partial}{\partial a_{4}}-c_{1} \frac{\partial}{\partial c_{1}}+c_{2} \frac{\partial}{\partial c_{2}}+c_{3} \frac{\partial}{\partial c_{3}}-c_{4} \frac{\partial}{\partial c_{4}},
\end{aligned}
$$

as well as from the fact that

$$
Q d H_{0}=Q d K_{0}=Q d J_{1}=Q d J_{2}=0 .
$$


To show that (5.9) holds true is simply a matter of an explicit computation, while (5.10) follows from the definition of $Q$. In fact, the last two equations hold since $J_{1}$ and $J_{2}$ are Casimirs of $P^{\prime}$ invariant under $Z_{1}$ and $Z_{2}$. For, e.g., $H_{0}$ one computes

$$
\begin{aligned}
Q d H_{0} & =P^{\prime} d H_{0}-R d H_{0}=X_{1}^{1}-\sum_{i, j=1}^{2}\left(G^{-1}\right)_{i j} L_{Z_{j}}\left(H_{0}\right) \cdot X_{1}^{i} \\
& =X_{1}^{1}-\sum_{i, j=1}^{2}\left(G^{-1}\right)_{i j} G_{j 1} \cdot X_{1}^{i}=X_{1}^{1}-\sum_{i} \delta_{i 1} \cdot X_{1}^{i}=0
\end{aligned}
$$

where the second equality follows from the fact that all the functions $H_{i}, K_{\alpha}, J_{\alpha}$ are in involution with respect to $P$.

Thanks to the above lemma, the generic symplectic leaf $\mathcal{S}_{\boldsymbol{\kappa}}$ is endowed with the structure of a regular bihamiltonian manifold. We know from Section 4 that the non trivial Hamiltonians $H_{1}, H_{2}, H_{3}, K_{1}$ (more precisely, the restriction to $\mathcal{S}_{\boldsymbol{\kappa}}$ of these Hamiltonians) satisfy the hypothesis of Theorem 3.1 with respect to the (restriction to $\mathcal{S}_{\boldsymbol{\kappa}}$ ) of the pencil $Q-\lambda P$. This fact can be directly shown as follows:

$$
Q d H_{i}=P^{\prime} d H_{i}-\sum_{i, j=1}^{2}\left(G^{-1}\right)_{i j}\left(Z_{i} \wedge X_{1}^{j}\right)\left(d H_{i}\right)=P d H_{i+1}-\sum_{i, j=1}^{2}\left(G^{-1}\right)_{i j} L_{Z_{i}}\left(H_{i}\right) X_{1}^{j}
$$

(where we understand $H_{4}=0$ ) and

$$
Q d K_{2}=P^{\prime} d K_{1}-\sum_{i, j=1}^{2}\left(G^{-1}\right)_{i j}\left(Z_{i} \wedge X_{1}^{j}\right)\left(d K_{2}\right)=\sum_{i, j=1}^{2}\left(G^{-1}\right)_{i j} L_{Z_{i}}\left(d K_{1}\right) X_{1}^{j}
$$

So we proved that, for generic values $\kappa_{i}$, with $i=1, \ldots, 4$, of the Casimirs, the system obtained by restriction of the Toda ${ }_{3}^{4}$ flows on $\mathcal{S}_{\kappa}$ is separable in the DN coordinates associated with the restriction to $\mathcal{S}_{\boldsymbol{\kappa}}$ of the pencil $Q-\lambda P$. To finish our job we finally have to:

a) explicitly compute the DN coordinates;

b) find the separation relations.

To solve the first problem, we will use the tools briefly described in Subsection 2.2. We rely on a result of [21], as well as on explicit computations, to state the following proposition, whose first part has been already discussed in Section 4.

Proposition 5.4 Let us consider the matrix

$$
\mathcal{G}(\lambda)=\left(\begin{array}{ll}
L_{Z_{1}} H(\lambda) & L_{Z_{2}} H(\lambda) \\
L_{Z_{1}} K(\lambda) & L_{Z_{2}} K(\lambda)
\end{array}\right)
$$


The roots of the degree-4 polynomial $\operatorname{Det}(\mathcal{G}(\lambda))$ are the roots of the minimal polynomial $\Delta(\lambda)=\lambda^{4}-\sum_{i=1}^{4} p_{i} \lambda^{4-i}$ of the Nijenhuis tensor $N=P^{-1} Q$ associated with the regular Poisson pencil $Q_{\lambda}$. The coefficients $p_{i}$ are functionally independent on the generic symplectic leaf $\mathcal{S}_{\kappa}$. Furthermore, the ratios

$$
\rho(\lambda)=-\mathcal{G}_{22} / \mathcal{G}_{12}, \quad \sigma(\lambda)=-\mathcal{G}_{21} / \mathcal{G}_{11}
$$

are Nijenhuis function generators.

Thus, one half of the Darboux-Nijenhuis coordinates will be given by the roots of $\operatorname{Det}(\mathcal{G}(\lambda))$. To find the remaining half we consider the vector field $Y=-P d p_{1}$, whose role has been discussed in Subsection 2.2. Since an explicit computations shows that $L_{Y} \log (\rho(\lambda))=1$, we can state the following

Proposition 5.5 A set of Darboux-Nijenhuis coordinates for the restriction to the generic symplectic leaf $\mathcal{S}_{\boldsymbol{\kappa}}$ of the Toda $a_{3}^{4}$ flows are given by the four roots $\lambda_{i}$ of $\operatorname{Det}(\mathcal{G}(\lambda))$ and by the values $\mu_{i}$ of the function $\log (\rho(\lambda))$ for $\lambda=\lambda_{i}$, where

$$
\rho(\lambda)=\frac{\left(-c_{1} a_{3}-a_{1} c_{2}\right) \lambda+c_{2} a_{1} b_{3}-a_{1} a_{2} a_{3}+c_{1} b_{2} a_{3}+c_{1} c_{2}}{c_{1} c_{3} \lambda+a_{1} a_{2} c_{3}-c_{1} b_{2} c_{3}} .
$$

(We assume that $c_{3} \neq 0$ and $c_{1} \lambda_{i}+a_{1} a_{2}-b_{2} c_{1} \neq 0$ for all $i=1, \ldots, 4$.)

To find the separation relations, we notice that the pairs $\left(\lambda_{i}, \rho\left(\lambda_{i}\right)\right)$ are common solutions to the system

$$
\left\{\begin{array}{l}
\rho \mathcal{G}_{11}+\mathcal{G}_{21}=0 \\
\rho \mathcal{G}_{12}+\mathcal{G}_{22}=0
\end{array}\right.
$$

since the rank of $\mathcal{G}\left(\lambda_{i}\right)$ is equal to 1 . Then we reconsider the Lax matrix (5.4), and we compute the Lie derivatives of the matrix $\mathcal{L}(\lambda, \nu)=\lambda \mathbf{1}-L(\nu)$ along the vector fields $Z_{i}$ :

$$
L_{Z_{1}}(\mathcal{L}(\lambda, \nu))=\left[\begin{array}{cccc}
0 & 0 & 0 & 0 \\
0 & 0 & 0 & 0 \\
0 & 0 & 0 & 0 \\
0 & 0 & 0 & 1
\end{array}\right], \quad L_{Z_{2}}(\mathcal{L}(\lambda, \nu))=\left[\begin{array}{cccc}
0 & 0 & 0 & -\nu^{-1} \\
0 & 0 & 0 & 0 \\
0 & 0 & 0 & 0 \\
0 & 0 & 0 & 0
\end{array}\right]
$$

Since

$$
\left.L_{Z_{a}}[\operatorname{Det}(\mathcal{L}(\lambda, \nu))]=\operatorname{Tr}\left[L_{Z_{a}}(\mathcal{L}(\lambda, \nu)) \mathcal{L}(\lambda, \nu)^{\vee}\right)\right], \quad a=1,2,
$$

$\mathcal{L}(\lambda, \nu)^{\vee}$ being the classical adjoint to $\mathcal{L}(\lambda, \nu)=\lambda \mathbf{1}-L(\nu)$, it follows from Proposition 5.2 and the definition (5.11) of $\mathcal{G}(\lambda)$ that the solutions of the system (5.12) are related to those of the system

$$
\left\{\begin{array}{l}
{\left[\mathcal{L}(\lambda, \nu)^{\vee}\right]_{44}=0} \\
{\left[\mathcal{L}(\lambda, \nu)^{\vee}\right]_{41}=0}
\end{array}\right.
$$

via $\rho=\nu^{4}$. Now we can state 
Proposition 5.6 The separation relations connecting pairs of Darboux-Nijenhuis coordinates $\left(\lambda_{i}, \mu_{i}\right)$, the Hamiltonians $H_{1}, H_{2}, H_{3}, K_{2}$, and the Casimirs $H_{0}, K_{1}, K_{2}, J_{0}$ are, on the generic symplectic leaf $\mathcal{S}_{\boldsymbol{\kappa}}$, given by the evaluation of the characteristic polynomial $\operatorname{Det}(\mathcal{L}(\lambda, \nu))$ in $\lambda=\lambda_{i}$ and $\nu=\nu_{i}=\exp \left(\frac{\mu_{i}}{4}\right)$.

Proof. We know that the pairs $\left(\lambda_{i}, \nu_{i}\right)$ solve system (5.1), and we have to show that they satisfy $\operatorname{Det}\left(\mathcal{L}\left(\lambda_{i}, \nu_{i}\right)\right)=0$. This can be done with the following adaptation of Sklyanin's technique, concerning the poles of the (normalized) Baker-Akhiezer function. Let us consider the $5 \times 4$ matrix $\mathcal{M}_{i}$ obtained by putting the vector $(0,0,0,1)$ on the top of $\mathcal{L}\left(\lambda_{i}, \nu_{i}\right)$. As we have assumed at the end of Proposition 5.5, the $3 \times 3$ matrix extracted from $\mathcal{M}_{i}$ by removing the 3 rd column and the 2 nd and the 4 th rows, is invertible. Since the system (5.1) is satisfied, the rank of $\mathcal{M}_{i}$ is 3 , and therefore $\operatorname{Det}\left(\mathcal{L}\left(\lambda_{i}, \nu_{i}\right)\right)$ vanishes.

We notice that, a posteriori, the separation coordinates for the $\operatorname{Toda}_{3}^{4}$ system fall in the class described in, e.g., [40, 1, 13, 41, 2, 29]. Namely, the DN coordinates that separate the Toda ${ }_{3}^{4}$ system are algebro-geometrical Darboux coordinates associated with the spectral curve (5.6), and fulfill the so-called Sklyanin's 'magic recipe'. This is a quite general fact, as discussed in Section 4 (see also [42] and Remark 2.12).

As a final remark, in connection with the discussion on the relation between the bihamiltonian property of an integrable vector field and the separability of the associated HJ equations of Section 3, we notice that the Hamiltonians $H_{1}, H_{2}, H_{3}, K_{2}$ are functionally independent from the coefficients of the minimal polynomial of the Nijenhuis tensor obtained from $Q-\lambda P$. So, this is a further instance of a system which is not bihamiltonian on a regular manifold, but turns out to be separable via the bihamiltonian method of SoV.

\subsection{A remarkable subsystem: the open $\operatorname{Toda}_{3}^{4}$ system}

In this last subsection we will discuss a remarkable reduction of the periodic Toda 4 system (even though less interesting from the point of view of algebraic integrability), leading to the corresponding generalization of the open (or non-periodic) one. In the manifold $M \simeq \mathbb{C}^{12}$ we consider the nine-dimensional submanifold $M_{0}$ defined by the equations

$$
a_{4}=c_{3}=c_{4}=0 .
$$

One can easily verify that the restriction $X_{H_{G T}}^{0}$ to $M_{0}$ of the vector field $X_{H_{G T}}$ is tangent to $M_{0}$. Also, the tensor $P$ can be restricted to $M_{0}$; indeed, the expression of its restriction $P_{0}$ with respect to the natural coordinates $\left\{b_{1}, \ldots, b_{4}, a_{1}, \ldots, a_{3}, c_{1}, c_{2}\right\}$ of $M_{0}$ is obtained from (5.2) simply by removing the 9 th, 11th, 12 th rows and columns. Moreover, one can check that $X_{H_{G T}}^{0}=P_{0} d H_{G T, 0}$, with

$$
H_{G T, 0}=\frac{1}{2}\left(b_{1}^{2}+b_{2}^{2}+b_{3}^{2}+b_{4}^{2}\right)-\left(a_{1}+a_{2}+a_{3}\right),
$$


and recognize that this function is the Hamiltonian of the open Toda lattice. Also, a Lax pair for $X_{H_{G T}}^{0}$ is

$$
L_{0}=\left[\begin{array}{cccc}
b_{1} & -\nu & 0 & 0 \\
\frac{a_{1}}{\nu} & b_{2} & -\nu & 0 \\
\frac{c_{1}}{\nu^{2}} & \frac{a_{2}}{\nu} & b_{3} & -\nu \\
-\nu & \frac{c_{2}}{\nu^{2}} & \frac{a_{3}}{\nu} & b_{4}
\end{array}\right], \quad \Phi_{0}=\left[\begin{array}{cccc}
0 & 0 & 0 & 0 \\
\frac{a_{1}}{\nu} & 0 & 0 & 0 \\
\frac{c_{1}}{\nu^{2}} & \frac{a_{2}}{\nu} & 0 & 0 \\
0 & \frac{c_{2}}{\nu^{2}} & \frac{a_{3}}{\nu} & 0
\end{array}\right] .
$$

It should be clear from the form of the Lax pair that the system associated to the vector field $X_{H_{G T}}^{0}$ is an extension of the standard open Toda lattice towards the so-called full open Toda lattice, which is a system describing a flow on the lower Borel subgroup of $\operatorname{sl}(N)$. The integrability of the full (open) Toda lattice was established in [12] (see, also, $[15])$. The idea was to complement the integrals of the motion coming from the Lax representation with additional integrals obtained by means of the 'chopping method', within the group-theoretical point of view.

The need to supply the standard results of the Lax theory with further methods should be clear from the following considerations. The only Casimir function of $P_{0}$ is $h_{0}=\sum_{i=1}^{4} b_{i}$. Hence, its symplectic leaves $\mathcal{S}_{\xi} \subset M_{0}$ are the eight-dimensional manifolds defined by $h_{0}=\xi$, and $X_{H_{G T}}^{0}$ can be seen as a Hamiltonian system with four degrees of freedom. The characteristic polynomial of the matrix $L_{0}$ is

$$
\operatorname{Det}\left(\lambda \mathbf{1}-L_{0}(\nu)\right)=-\nu^{4}+\lambda^{4}-h_{0} \lambda^{3}+h_{1} \lambda^{2}-h_{2} \lambda+h_{3},
$$

that is, it provides us with only three non trivial Hamiltonians,

$$
\begin{aligned}
& h_{1}=\sum_{i>j=1}^{4} b_{i} b_{j}+\sum_{i=1}^{3} a_{i}, \quad h_{2}=\sum_{i>j>k=1}^{4} b_{i} b_{j} b_{k}+\sum_{i=1}^{3} a_{i}\left(b_{i+2}+b_{i+3}\right)+c_{1}+c_{2} \\
& h_{3}=b_{1} a_{2} b_{4}+a_{1} b_{3} b_{4}+b_{1} b_{2} a_{3}+b_{1} c_{2}+a_{1} a_{3}+c_{1} b_{4}+b_{1} b_{2} b_{3} b_{4} .
\end{aligned}
$$

We will now show how the tools we previously introduced can be used to geometrically prove the complete integrability of such a system and, moreover, yield the existence of an additional integral of the motion. The main property is that, along with $P$, the tensor $Q$ restricts to $M_{0}$. This can be proven as follows: one checks by direct inspection that this is true for $P^{\prime}$; then the assertion follows from the fact that the vector field $X_{1}^{2}=P^{\prime} d K_{0}$ vanishes on $M_{0}$, while $Z_{1}$ and $X_{1}^{1}$, which coincides with $X_{G T}$, are tangent to $M_{0}$.

Furthermore, we add two observations. The first one concerns the restriction $\mathcal{G}_{0}$ to $M_{0}$ of the matrix $\mathcal{G}$. It has the form

$$
\mathcal{G}_{0}=\left[\begin{array}{cc}
\mathcal{G}_{11}^{0} & \lambda^{2}-\left(b_{2}+b_{3}\right) \lambda+b_{2} b_{3}+a_{2} \\
0 & -\left(c_{1} a_{3}+a_{1} c_{2}\right) \lambda-a_{1} a_{2} a_{3}+c_{1} c_{2}+c_{1} b_{2} a_{3}+a_{1} c_{2} b_{3}
\end{array}\right]
$$

and therefore its determinant (that is, the minimal polynomial of the Nijenhuis tensor $N_{0}$ induced by the pencil $Q_{0}-\lambda P_{0}$ on $\mathcal{S}_{\xi}$ ) factors as $\mathcal{G}_{11}^{0} \mathcal{G}_{22}^{0}$, where $\mathcal{G}_{11}^{0}=\lambda^{3}-\pi_{1} \lambda^{2}-\pi_{2} \lambda-\pi_{3}$ 
is a degree-three polynomial. The second observation consists in the fact that the three surviving Hamiltonians $h_{1}, h_{2}, h_{3}$ given by (5.17) satisfy the conditions:

$$
Q_{0} d h_{i}=\sum_{j=1}^{3} F_{i j}^{0} P_{0} d h_{i}, \text { with } F_{i j}^{0}=\left[\begin{array}{ccc}
\pi_{1} & 1 & 0 \\
\pi_{2} & 0 & 1 \\
\pi_{3} & 0 & 0
\end{array}\right] .
$$

We notice that the functions $\pi_{1}, \pi_{2}, \pi_{3}$, and the root

$$
\lambda_{4}=\frac{-a_{1} a_{2} a_{3}+c_{1} c_{2}+a_{1} c_{2} b_{3}+c_{1} b_{2} a_{3}}{c_{1} a_{3}+a_{1} c_{2}}
$$

of $\mathcal{G}_{22}^{0}$ are still functionally independent and hence (generically) different on $\mathcal{S}_{\xi}$.

Lemma 5.7 Let $\sigma$ be any function satisfying $Q_{0} d \sigma=\lambda_{4} P_{0} d \sigma$. Under the above hypotheses, the brackets $\left\{\sigma, h_{i}\right\}_{P_{0}}$ and $\left\{\sigma, h_{i}\right\}_{Q_{0}}$ vanish.

Proof. Evaluating both sides of $Q_{0} d \sigma=\lambda_{4} P_{0} d \sigma$ on the differentials $\left(d h_{1}, d h_{2}, d h_{3}\right)$, and switching the action of the Poisson tensors on the $d h_{i}$ 's, we get

$$
\left\langle d \sigma, Q_{0} d h_{i}\right\rangle=\lambda_{4}\left\langle d \sigma, P_{0} d h_{i}\right\rangle, \quad i=1,2,3 .
$$

Inserting (5.19) we get the equation $\sum_{j=1}^{3}\left(F_{i j}^{0}-\lambda_{4} \delta_{i j}\right)\left\langle d \sigma, P_{0} d h_{j}\right\rangle=0$. Since $\lambda_{4}$ is not an eigenvalue of $F_{i j}^{0}$, the lemma is proved.

So a fourth integral of the motion, that commutes with the Hamiltonian $H_{G T}^{0}$ of the open Toda ${ }_{3}^{4}$ lattice, is given indeed by the distinguished root $\lambda_{4}$ of equation (5.20); this constructively proves the integrability of the system.

Finally, we notice that this method proves the existence of a fifth integral of the motion. Indeed, we know that, along with $\lambda_{4}$, there must exist another independent function $\mu_{4}$, satisfying the hypotheses of Lemma 5.7 and functionally independent of $\lambda_{4}$ and of the $h_{i}$ 's. In such a comparatively low dimensional case, such a function can be explicitly found to be

$$
\mu_{4}=\frac{c_{2}\left(a_{1} b_{2} c_{1}-a_{2} a_{1}^{2}-c_{1}^{2}-c_{1} b_{3} a_{1}\right)}{c_{1}\left(c_{1} a_{3}+a_{1} c_{2}\right)\left(\lambda_{4}^{3}-\pi_{1} \lambda_{4}{ }^{2}-\pi_{2} \lambda_{4}-\pi_{3}\right)} .
$$

\section{Acknowledgments}

We wish to thank Franco Magri for stimulating our interest in the theory of bihamiltonian manifolds, and, in particular for sharing with us his insights about the problem of SoV. The results presented in Sections 2 and 3 have been obtained in a long-standing joint-work with him. We are also grateful to Boris Dubrovin and Giorgio Tondo for a number of useful discussions. Some computations were performed with Maple $V^{\circledR}$. 


\section{References}

[1] Adams, M.R., Harnad, J., and Hurtubise, J., Darboux coordinates and LiouvilleArnold integration in Loop Algebras, Commun. Math. Phys., 1993, 155, pp. 385413.

[2] Adams, M.R., Harnad, J., and Hurtubise, J., Darboux coordinates on coadjoint orbits of Lie Algebras, Lett. Math. Phys., 1997, 40, pp. 41-57.

[3] Adler, M., van Moerbeke, P., and Vanhaecke, P., Algebraic integrability, Painlevé geometry and Lie algebras, Springer-Verlag, Berlin, 2004.

[4] Arnol'd, V.I., Mathematical methods of classical mechanics, Springer, 1989.

[5] Bartocci, C., Falqui, G., and Pedroni, M., A geometric approach to the separability of the Neumann-Rosochatius system, Differential Geom. Appl., 2004, 21, no. 3, pp. 349-360.

[6] Benenti, S., Intrinsic characterization of the variable separation in the HamiltonJacobi equation, J. Math. Phys. 1997, 38, no. 12, pp. 6578-6602.

[7] Błaszak, M., Bi-Hamiltonian separable chains on Riemannian manifolds, Phys. Lett. A, 1998, 243, pp. 25-32.

[8] Błaszak, M., Theory of separability of multi-Hamiltonian chains, J. Math. Phys., 1999, 40, pp. 5725-5738.

[9] Brouzet, R., Caboz, R., Rabenivo, J., and Ravoson, V., Two degrees of freedom quasi-bihamiltonian systems, J. Phys. A: Math. Gen., 1996, 29, pp. 2069-2076.

[10] Damianou, P.A., Magri, F., A gentle (without chopping) approach to the full Kostant-Toda lattice, SIGMA Symmetry Integrability Geom. Methods Appl., 2005, 1, Paper 010, 12 pp. (electronic).

[11] Degiovanni, L., and Magnano, G., Tri-hamiltonian vector fields, spectral curves, and separation coordinates, Rev. Math. Phys., 2002, 14, pp. 1115-1163.

[12] Deift, P.A., Li, L.-C., Nanda, T., and Tomei, C., The Toda lattice on a generic orbit is integrable, Comm. Pure Appl. Math., 1984, 39, pp. 183-232.

[13] Dubrovin, B.A., and Diener, P., Algebraic geometrical Darboux coordinates in $R$ matrix formalism, 1994, SISSA preprint 88/94/FM.

[14] Dubrovin, B.A., Krichever, I.M., and Novikov, S.P., Integrable Systems I. In: Enc. of Math. Sciences, vol. 4, Springer Verlag, Berlin, 1990. 
[15] Ercolani, N.M., Flaschka, H., and Singer, S., The geometry of the full Kostant-Toda lattice. In: Integrable systems: the Verdier Memorial Conference (O. Babelon et al., eds.), Progress in mathematics, vol. 115, Birkhäuser, Boston, 1993, pp. 181-226.

[16] Falqui, G., Magri, F., Pedroni, M., and Zubelli, J.-P., A Bi-Hamiltonian Theory for Stationary KdV Flows and their Separability, Regul. Chaotic Dyn., 2000, 5, pp. $33-51$.

[17] Falqui, G., Magri, F., and Tondo, G., Bihamiltonian systems and separation of variables: an example from the Boussinesq hierarchy, Theor. Math. Phys., 2000, 122, pp. 176-192.

[18] Falqui, G., and Musso, F., Gaudin Models and Bending Flows: a geometrical point of view, J. Phys. A: Math. Gen., 2003, 36, pp. 11655-11676.

[19] Falqui, G., and Musso, F., On Separation of Variables for Homogeneous SL(r) Gaudin Systems, Math. Phys. Anal. Geom., 2006, 9, pp. 233-262.

[20] Falqui, G., and Pedroni, M., On a Poisson Reduction for Gel'fand-Zakharevich Manifolds, Rep. Math. Phys., 2002, 50, pp. 395-407.

[21] Falqui, G., and Pedroni, M., Separation of variables for bi-Hamiltonian systems, Math. Phys. Anal. Geom., 2003, 6, pp. 139-179.

[22] Falqui, G., and Pedroni, M., Gel'fand-Zakharevich systems and algebraic integrability: the Volterra lattice revisited, Regul. Chaotic Dyn., 2005, 10, pp. 399-412.

[23] Gel'fand, I.M., and Zakharevich, I., On the local geometry of a bi-Hamiltonian structure. In: The Gel'fand Mathematical Seminars 1990-1992 (L. Corwin et al., eds.), Birkhäuser, Boston, 1993, pp. 51-112.

[24] Gel'fand, I.M., and Zakharevich, I., Webs, Lenard schemes, and the local geometry of bihamiltonian Toda and Lax structures, Selecta Math. (N.S.), 2000, 6, pp. 131183.

[25] Harnad, J., and Hurtubise, J., Multi-Hamiltonian structures for r-matrix systems, J. Math. Phys., 2008, 49, no. 6, 062903, 21 pp.

[26] Hurtubise, J., Separation of variables and the geometry of Jacobians, SIGMA Symmetry Integrability Geom. Methods Appl., 2007, 3, Paper 017, 14 pp.

[27] Ibort, A., Magri, F., and Marmo, G., Bihamiltonian structures and Stäckel separability, J. Geom. Phys., 2000, 33, pp. 210-228.

[28] Kupershmidt, B.A., Discrete Lax equations and differential-difference calculus, Asterisque, 1985, 123, pp. 212-245. 
[29] Kuznetsov, V.B., Nijhoff, F.W., and Sklyanin, E.K., Separation of variables for the Ruijsenaars system, Commun. Math. Phys., 1997, 189, pp. 855-877

[30] Magri, F., Geometry and soliton equations. In: La Mécanique Analytique de Lagrange et son héritage, Atti Acc. Sci. Torino Suppl., 1990, 124, pp. 181-209.

[31] Magri, F., Falqui, G., and Pedroni, M., The method of Poisson pairs in the theory of nonlinear PDEs. In: Direct and inverse methods in nonlinear evolution equations, Lecture Notes in Phys., 632, Springer, Berlin, 2003, pp. 85-136.

[32] Magri, F., and Marsico, T., Some developments of the concept of Poisson manifolds in the sense of A. Lichnerowicz. In: Gravitation, electromagnetism, and geometrical structures (G. Ferrarese, ed.), Pitagora, Bologna, 1996, pp. 207-222.

[33] Magri, F., and Morosi, C., A geometrical characterization of integrable hamiltonian systems through the theory of Poisson-Nijenhuis manifolds, Quaderno S19/1984, 1984, Dipartimento di Matematica, Università di Milano.

[34] Marciniak, K., and Błaszak, M., Dirac reduction revisited, Jour. Nonlin. Math. Phys., 2003, 10, pp. 1-13.

[35] Marsico, T., Una caratterizzazione geometrica dei sistemi che ammettono rappresentazione alla Lax estesa, Ph. D. thesis, Università di Milano, 1996 (in Italian).

[36] Meucci, A., Toda equations, bihamiltonian systems, and compatible Lie algebroids, Math. Phys. Anal. Geom., 2001, 4, pp. 131-146.

[37] Morosi, C., and Tondo, G., Quasi-Bi-Hamiltonian systems and separability, J. Phys. A: Math. Gen., 1997, 30, pp. 2799-2806.

[38] Morosi, C., and Tondo, G., The quasi-Bi-Hamiltonian formulation of the Lagrange top, J. Phys. A: Math. Gen., 2002, 35, pp. 1741-1750.

[39] Pedroni, M., Bi-Hamiltonian aspects of the separability of the Neumann system, Theor. Math. Phys., 2002, 133, pp. 1720-1727.

[40] Sklyanin, E.K., Separations of variables in the classical integrable $S L(3)$ magnetic chain, Commun. Math. Phys., 1992, 150, pp. 181-192.

[41] Sklyanin, E.K., Separations of variables: new trends, Progr. Theor. Phys. Suppl., 1995, 118, pp. 35-60.

[42] Tsiganov, A.V., On the invariant separated variables, Regul. Chaotic Dyn., 2001, 6, no. 3, pp. 307-326.

[43] Vaisman, I., Lectures on the Geometry of Poisson Manifolds, Progress in Mathematics, Birkhäuser, Boston, 1996. 
[44] Veselov, A.P., and Novikov S.P., Poisson brackets and complex tori, Proceedings of the Steklov Math. Inst., 1985, 3, pp. 53-65.

[45] Weinstein, A., The local structure of a Poisson manifold, J. Diff. Geom., 1983, 18, pp. 523-557. (Errata and addenda, J. Diff. Geom., 1985, 22, p. 255). 